\title{
Lithium abundances of very low mass members of Chamaeleon I
}

\author{
C. M. S. Johnas ${ }^{1}$, E. W. Guenther ${ }^{2}$, V. Joergens ${ }^{3}$, A. Schweitzer ${ }^{1}$, and P. H. Hauschildt ${ }^{1}$ \\ 1 Hamburger Sternwarte, Gojenbergsweg 112, 21029 Hamburg, Germany \\ e-mail: cjohnas@hs.uni-hamburg.de \\ 2 Thüringer Landessternwarte Tautenburg, 07778 Tautenburg, Germany \\ 3 Max-Planck-Institut für Astronomie, Königstuhl 17, 69117 Heidelberg, Germany \\ Received 27 March 2007 / Accepted 6 August 2007
}

\begin{abstract}
Aims. We present the first study of the lithium abundances of very low mass objects in Chamaeleon I close to the hydrogen burning mass limit based on atmospheric models and high-resolution spectroscopic observations. The studied objects, $\mathrm{Cha} \mathrm{H} \alpha 2,3,4,5,6$ and 8 , are very young brown dwarf candidates and very low mass stars on the verge of lithium depletion.

Methods. For this analysis, we have computed a new "GAIA-cond" class model grid over effective temperatures from $2600 \mathrm{~K}$ to $3100 \mathrm{~K}$, surface gravities from $\log (g)=3.5$ to 5.5 , and lithium abundances from $\log \epsilon=0.0$ to 3.7 , for two different line profile setups introduced in previous work. Calculated synthetic spectra are compared with high-resolution UVES/VLT echelle spectra of the objects.

Results. We find good descriptions of the lithium resonance doublet lines at $6708 \AA$ and of the surrounding pseudo-continuum and determine a consistent set of lithium abundances $(\log (\epsilon)=1.55)$. However, the derived lithium abundances are lower than the meteoritic one $(\log (\epsilon)=3.31)$ and that of higher mass stars in Cha I $(\log (\epsilon)=3.1 / 3.4$ for LTE-/non-LTE-calculations $)$. By modeling the TiO-line, we demonstrate that veiling does not make the lithium lines appear weaker. We can also rule out that the results are spoiled by the presence of spots.

Conclusions. A possible explanation for these results would be that the objects are either more massive, or much older, than previously thought, so that the lithium depletion has already started. Although the uncertainties of the masses and ages are large, they are not large enough as to explain the observed lithium depletion. Therefore, the most likely explanation is either a lack of understanding of the details of the formation of the lithium line, or a lack of understanding of the internal structure of the very young low-mass objects.
\end{abstract}

Key words. stars: atmospheres - line: profiles - stars: low-mass, brown dwarfs - stars: pre-main sequence - ISM: individual objects: Chamaeleon I

\section{Introduction}

Brown dwarfs (BDs) are objects that are not massive enough to sustain thermonuclear fusion of hydrogen at their centers but are distinguished from gas-giant planets by their ability to burn deuterium. Thermonuclear fusion reactions in young stars do not only include the burning of hydrogen but also of heavier elements, for example Li I, above very similar mass limits. Since lithium is quickly depleted in young stars, the presence of an original amount of Li I in a number of old, very low-mass objects, together with the assumption of fully convective interiors in this mass regime, has been used as an argument that these objects fuse neither lithium nor hydrogen and are thus BDs. Therefore, the Li I abundance is a very important diagnostic for identifying old BDs ("Lithium-test") (Rebolo et al. 1992; Basri 2000). The application of this substellar test is more complicated for very massive BDs and very young stars (ages of a few millions years). Massive BDs (above $0.06 M_{\odot}$ ) burn lithium: they can even burn hydrogen, however not at a rate sufficient to fully compensate radiative losses. Young stars with an age of about one Myr or less also show a substantial amount of Li I in their spectra because they are too young to have burned their initial lithium, i.e., their central temperature has not yet reached the lithium burning limit. In order to better understand the Li I

* Based on observations obtained at the European Southern Observatory at Paranal, Chile in program 65.L-0629(A,B) and 65.I-0011(A). depletion in young objects, it is required to study the abundance of Li I in very young (a few Myr) objects at the boundary between stars and BDs, and to compare it with the Li I abundance of higher mass stars of similar age.

The determination of element abundances from spectroscopic observations of such low-mass and, hence, cool objects, such as BDs, is hampered by the large number of absorption lines in their spectra leading to the fact that there is essentially no continuum in the optical regime. It is therefore impossible to completely identify the Li I resonance absorption line from observations alone (this is valid for any other spectral line of objects in this effective temperature regime). A way out is to calculate synthetic spectra of the objects, including all spectral lines, and fit them to observed spectra by varying the abundance of Li I. This requires a sophisticated modeling of the (sub)stellar atmosphere. We have generated a grid of model atmospheres and spectra with varied lithium abundances with the general purpose stellar atmosphere code PHOENIX (Hauschildt \& Baron 1999) using two different setups for the lithium absorption line profile treatment (Johnas et al. 2006). In addition to work by members of the PHOENIX group, synthetic spectra of BDs that aim at an improved description of the lithium resonance lines have also been calculated previously by Pavlenko et al. (2000) and Tsuji (2001).

Current observational studies of lithium in BD atmospheres have been performed by means of measurements of the equivalent width $(E W)$ of Li I based on high-resolution spectra by 


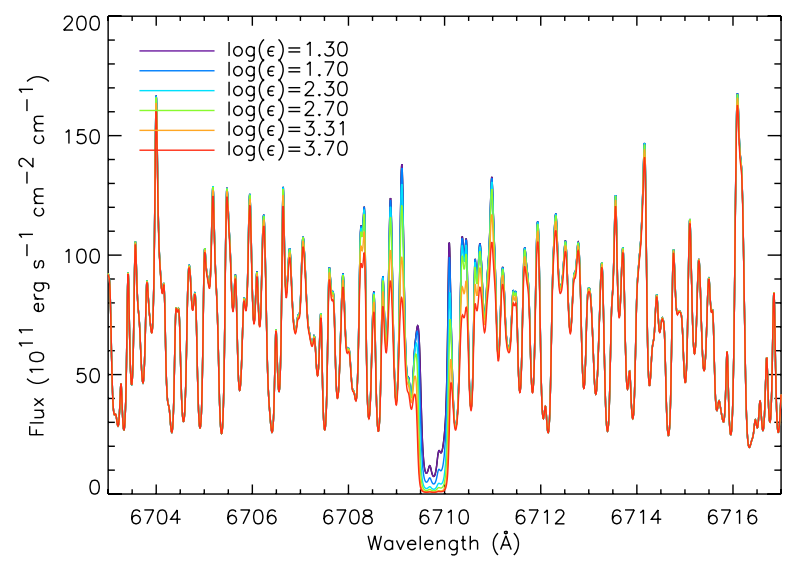

Fig. 1. Synthetic spectra of Setup2 for a $T_{\text {eff }}=3000 \mathrm{~K}$ and $\log (g)=4.0$ displaying the Li I with varied abundances from $\log (\epsilon)=1.3$ to 3.7 .

Tinney (1998), Joergens \& Guenther (2001), and Pavlenko (2005), based on intermediate-resolution spectra by Kenyon et al. (2005) and Neuhäuser \& Comerón (1999), and based on low-resolution spectra by Zapatero Osorio et al. (2002).

However, as mentioned before, due to the large number of densely spaced spectral lines in BD and very low-mass star (VLMS) spectra and the missing continuum, the determination of the lithium abundances via a derived $E W$ and comparison with theoretical curves of growth implicates large uncertainties and should be considered with care. Of course, the missing continuum also has an influence on the determination of the rotational broadening. The approximation of the continuum influences the results of the $E W \mathrm{~s}$. In standard methods the continuum is approximated by a straight line, which is not sufficient and does not provide reliable values of the derived $E W \mathrm{~s}$. It is important to have a description of the continuum as close as possible, e.g., synthetic pseudo continua. Exemplarily, a theoretical series of synthetic spectra of Setup2, see Sect. 3, in a representative combination of the stellar parameters, $T_{\text {eff }}=3000 \mathrm{~K}$ and $\log (g)=4.0$, showing the Li I line is presented in Fig. 1, in which the challenges mentioned above are visible. In all previous studies of these objects, the EWs and the rotational broadening were determined by fitting a continuum to the data. Since we model the entire spectrum in this work, we avoid the problems associated with continuum fitting and methods using $E W s$ and curves of growth. Thus, the new values of the $E W \mathrm{~s}$ and rotational broadening differ from the older ones. In this work we present the results of a Li I abundance study of very low mass members of the Chamaeleon I star forming region. The aim of this work is to better understand the Li I depletion in young objects close to the boundary between stars and BDs. Detailed studies have led to the identification of a number of BDs and VLMSs in the Chamaeleon I region (Comerón et al. 1999, 2000; Luhman 2004). Among them are the BD candidates $\mathrm{Cha} \mathrm{H} \alpha 2,3$, 6 and 8 (spectral type M6.5-M7) (Comerón et al. 2000) and the VLMSs Cha H $\alpha 4$ and 5 (M6) (Comerón et al. 2000), for which high-resolution spectra have been taken by Joergens \& Guenther (2001) and Joergens (2006), and whose Li I abundances will be discussed in detail in this work. An overview of the derived stellar properties from Comerón et al. (1999, 2000) and Luhman (2004) can be found in Table 1 . The varying values in the tables represent the uncertainties in the stellar properties when studying these objects. These objects cannot currently be identified unambiguously as either young stars or BDs. Note that due to differences in the calibrations Neuhäuser \& Comerón (1999) considered Cha $\mathrm{H} \alpha 3$ and 6 as bona fide BDs. Comerón et al. (2000) derived masses for Cha $\mathrm{H} \alpha 2$, 3, 4, 5, 6 and 8 between $0.05 M_{\odot}$ and $0.11 M_{\odot}$, and ages between $<2$ and $3 \mathrm{Myr}$ based on evolutionary models of Baraffe et al. (1998). These age estimates are in agreement with a determination of the mean age of the Chamaeleon I association (3 Myr) by López Martí et al. (2004). The determination of the effective temperature and luminosity of the objects is crucial when applying evolutionary track calculations in order to give an estimate of the mass and age of the objects. Applying different sets of evolutionary tracks leads to (slightly) different masses and ages, see Table 1. For example, in Comerón et al. (2000), evolutionary models by Burrows et al. (1997) yield slightly higher masses and a factor of two greater ages compared to the predictions by track calculations from Baraffe et al. (1998). These discrepancies are due to uncertainties in evolutionary models for such small ages and masses, e.g., due to the fact that these objects are still contracting, missing calibrations of the models with observations, and the difficulty of setting the initial conditions of the models. Nevertheless, the cores of these objects either never reach the lithium burning temperature (when considering the models of Baraffe et al. 1998) or are only on the verge of reaching it (when considering the results of the Burrows et al. 1997, models) (Chabrier \& Baraffe 2000).

\section{Observations}

We use two sets of observations in this paper. First, there are high-resolution spectroscopic observations of the BDs/VLMSs Cha $\mathrm{H} \alpha 2,3,4,5,6$ and 8, which have been carried out with the Uv-Visual Echelle Spectrograph (UVES) at the VLT Unit telescope 2 (KUEYEN) by Joergens \& Guenther (2001). They have been subjected to an improved data reduction by Joergens (2006), which is consistent for the lithium resonance line with the previous method. The IRAF Echelle package was used to subtract the bias, flat-field and extract the spectra, remove the scattered light, cosmic rays and the sky background, and finally to wavelength calibrate the spectra. Details of the reduction and wavelength calibration can be found in the given references. We note here only that no rebinning of the spectra was done in order to avoid any changes of the line profiles. In this work, we use between 1 and 7 individual spectra for each object depending on availability and signal-to-noise ratio. We label the spectra of each Cha I object with the modified Julian day (MJD) of its observation, as defined by MJD $=\mathrm{JD}-2400000.5$. The obtained spectra cover simultaneously the wavelength regions from $6700 \AA$ to $8545 \AA$ and $8640 \AA$ to $10400 \AA$, at a resolution of $R=40000$. The Li I doublet line at $6708 \AA$ is in the last order of the echelle spectrum, which is only partly covered by the detector. Nevertheless, it is possible to extract the line and the continuum red-wards of it.

In the second set of observations, we use spectra of Cha $\mathrm{H} \alpha$ 2, 3, 5 and 6, which have been published by Natta et al. (2004). They were also obtained with UVES at the same resolution of 40000 but with a different wavelength range (479-679 nm) covering both wings of the Li I doublet.

\section{Models and methods}

We have generated a model atmosphere grid with two different line profile setups: Setup1 corresponds to synthetic spectra with impact approximated van der Waals alkali line profiles (Schweitzer et al. 1996), whereas Setup2 represents synthetic 
Table 1. Stellar properties of Cha $\mathrm{H} \alpha$ objects.

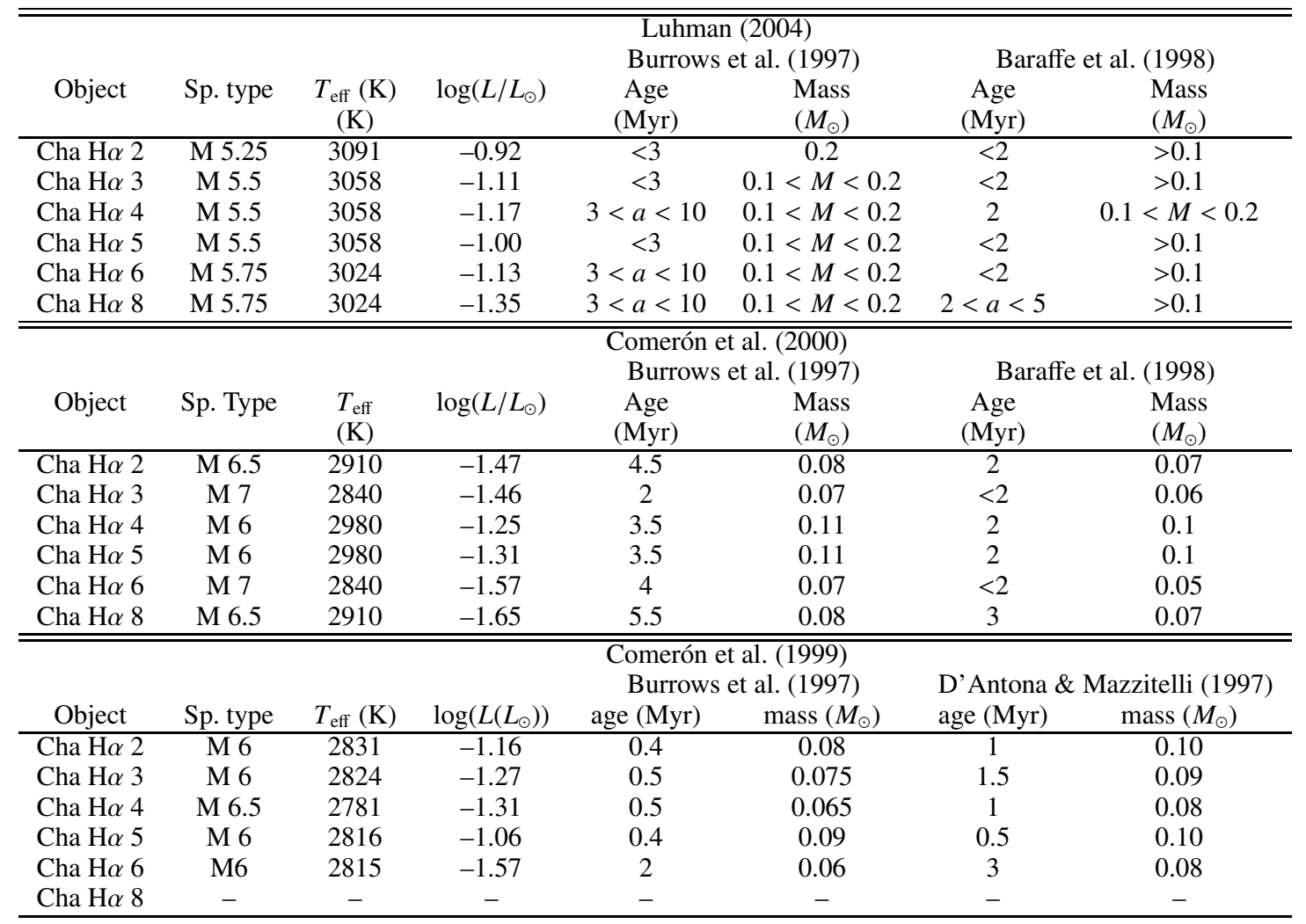

spectra with more sophisticated line profile calculations (Johnas et al. 2006; Allard et al. 2006, 2005). For both setups, models are calculated using varied lithium abundances. For each line profile setup, a model atmosphere is calculated and converged before generating the synthetic spectrum. "GAIA-cond" settings are applied for $T_{\text {eff }}=2600 \mathrm{~K}$ to $3100 \mathrm{~K}$ in steps of $100 \mathrm{~K}$. This temperature range covers the effective temperatures of the studied BDs/VLMSs as determined by Comerón et al. (2000) from spectral types. The "GAIA-cond" approximations consider fully "rained-out" condensed dust in chemical equilibrium, see Allard et al. (2001) for details. At these effective temperatures, dust is not important and the Cond-approximation can be applied. For calculating the model atmospheres, we have to assume a value for the surface gravity $\log (g)$. Since the masses are required in order to estimate the $\log (g)$-values, we have to use evolutionary tracks for the estimation. Using the table in Chabrier et al. (2000) for objects with an age of 1 and $5 \mathrm{Myr}$, and the luminosities of the objects derived by Luhman (2004) (see Table 1), we find $\log (g)$-values between 3.5 and $\geq 4$. For the luminosities derived by Comerón et al. (2000), we find values between 3.48 and 3.6 for an age of $1 \mathrm{Myr}$, and larger than 4 for an age of 5 Myr. Nevertheless, we want to stress that the luminosities in Table 1 also include the disk and accretion, which is why the luminosity of only the VLMS/BD is expected to be lower, and thus the values of $\log (g)$ higher. If we use the effective temperature instead of the luminosity, the masses of the objects are in general slightly higher and hence the $\log (g)$-values a bit larger. For this reason, we varied the surface gravity $\log (g)$ between 3.5 and 5.5 in steps of 0.5 . The lithium abundance is varied from $\log (\epsilon)=0.0$ to 3.7 in steps of 0.1 dex. For comparison, in standard spectra the meteoritic lithium abundance of $\log (\epsilon)=$ 3.31 is used. Every spectrum is calculated at a spectral resolution of 670000 for the wavelength range around the lithium resonance doublet. In the model atmosphere and synthetic spectra, we assume a microturbulence of $2.0 \mathrm{~km} \mathrm{~s}^{-1}$, which is a typical value used in $\mathrm{M}$ dwarf modeling (Allard \& Hauschildt 1995). A $\chi^{2}$-technique is applied to quantify the differences between observations and models, as described in Schweitzer et al. (2001). The synthetic spectra are first convolved down to the resolution of the observations $(R=40000)$. For the rotational broadening, a standard limb darkening coefficient is used (Gray 1992; Eqs. (17.11) and (17.12)).

The results of the fits are presented in Table 2 for the observations by Joergens \& Guenther (2001) and in Table 3 for the observations by Natta et al. (2004). The first row for each observation of each section is the fit over all dimensions of the parameter range. In order to limit the number of free parameters, the effective temperatures derived by Comerón et al. (2000) are used for a second fit. Results for this second fit are presented for each observation in the second row of Table 2.

The quality of the fit is very sensitive to the selected wavelength range. Therefore, we use the same range, from $6707 \AA$ to $6717 \AA$, in all our fits of the observations by Joergens \& Guenther (2001). The lower limit here is set by the wavelength range of the observations, which starts just shortward of $6707 \AA$. The small lithium $E W$ s obtained by Joergens \& Guenther (2001) justify the chosen range in wavelength. With the fit parameters obtained by this wavelength range, a satisfying description is also provided towards the redder part of the observations.

Although the $v \sin i$-values used for the modeling are in general agreement with the values given in Joergens \& Guenther (2001), there are noticeable differences in some cases. Notably, differences occur when the $E W$ s are determined by fitting a continuum to the spectral region close to the lithium line. Because of the large number of spectral lines in very late-type objects 
Table 2. Physical parameters of BDs/VLMSs in Chamaeleon I derived from a comparison of synthetic GAIA-cond models and high-resolution UVES spectra using different line profile setups: (from left to right) effective temperature, surface gravity, lithium abundance and $E W$ of lithium. The given results are for (i) a fit to the full range of free model parameters: $T_{\text {eff }}, \log (g), \log (\epsilon)$, radial velocity and rotational velocity (first row of each observation) and (ii) for a fit with fixed effective temperature as determined by Comerón et al. (2000) (second row).

\begin{tabular}{|c|c|c|c|c|}
\hline Object & $\begin{array}{l}T_{\text {eff }} \\
(\mathrm{K})\end{array}$ & $\log (g)$ & $\overline{\log (\epsilon)}$ & $\begin{array}{c}E W_{\mathrm{Li}} \\
(\AA)\end{array}$ \\
\hline \multicolumn{5}{|c|}{ Setupl } \\
\hline \multirow[t]{2}{*}{ Cha $\mathrm{H} \alpha 2, \mathrm{MJD}=51638.087$} & 3100 & 4.5 & 0.9 & 0.33 \\
\hline & 2900 & 3.5 & 1.0 & 0.34 \\
\hline \multirow{2}{*}{ Cha $\mathrm{H} \alpha 2, \mathrm{MJD}=51658.096$} & 3100 & 4.0 & 1.5 & 0.40 \\
\hline & 2900 & 3.5 & 1.6 & 0.41 \\
\hline \multirow[t]{2}{*}{ Cha $\mathrm{H} \alpha 2$ sum } & 3100 & 4.0 & 1.4 & 0.39 \\
\hline & 2900 & 3.5 & 1.4 & 0.39 \\
\hline \multirow[t]{2}{*}{ Cha $\mathrm{H} \alpha 3, \mathrm{MJD}=51638.987$} & 3100 & 4.0 & 1.0 & 0.34 \\
\hline & 2800 & 3.5 & 1.0 & 0.35 \\
\hline \multirow[t]{2}{*}{$\mathrm{Cha} \mathrm{H} \alpha 3, \mathrm{MJD}=51658.113$} & 3100 & 4.0 & 1.5 & 0.40 \\
\hline & 2800 & 3.5 & 1.5 & 0.41 \\
\hline \multirow[t]{2}{*}{ Cha $\mathrm{H} \alpha 3$ sum } & 3100 & 4.0 & 1.4 & 0.39 \\
\hline & 2800 & 3.5 & 1.3 & 0.39 \\
\hline \multirow[t]{2}{*}{ Cha $\mathrm{H} \alpha 4, \mathrm{MJD}=51617.210$} & 3100 & 4.0 & 1.8 & 0.44 \\
\hline & 3000 & 3.5 & 1.8 & 0.43 \\
\hline \multirow[t]{2}{*}{$\mathrm{Cha} \mathrm{H} \alpha$ 4, MJD = 51617.236} & 3100 & 4.0 & 1.8 & 0.44 \\
\hline & 3000 & 3.5 & 1.8 & 0.43 \\
\hline \multirow[t]{2}{*}{ Cha $\mathrm{H} \alpha 4, \mathrm{MJD}=51627.277$} & 3100 & 4.0 & 1.4 & 0.39 \\
\hline & 3000 & 3.5 & 1.5 & 0.40 \\
\hline \multirow[t]{2}{*}{ Cha $\mathrm{H} \alpha 4, \mathrm{MJD}=51627.303$} & 3100 & 4.0 & 1.4 & 0.39 \\
\hline & 3000 & 3.5 & 1.4 & 0.39 \\
\hline \multirow[t]{2}{*}{$\mathrm{Cha} \mathrm{H} \alpha 4, \mathrm{MJD}=51634.997$} & 3100 & 4.0 & 1.5 & 0.40 \\
\hline & 3000 & 3.5 & 1.6 & 0.41 \\
\hline \multirow[t]{2}{*}{$\mathrm{Cha} \mathrm{H} \alpha 4, \mathrm{MJD}=51635.023$} & 3100 & 4.0 & 1.6 & 0.41 \\
\hline & 3000 & 3.5 & 1.6 & 0.41 \\
\hline \multirow[t]{2}{*}{ Cha $\mathrm{H} \alpha 4, \mathrm{MJD}=51657.994$} & 3100 & 4.0 & 1.6 & 0.41 \\
\hline & 3000 & 3.5 & 1.6 & 0.41 \\
\hline \multirow[t]{2}{*}{ Cha $\mathrm{H} \alpha 4$ sum } & 3100 & 4.0 & 1.6 & 0.41 \\
\hline & 3000 & 3.5 & 1.6 & 0.41 \\
\hline \multirow[t]{2}{*}{ Cha $\mathrm{H} \alpha 5, \mathrm{MJD}=51639.009$} & 3100 & 4.5 & 1.6 & 0.43 \\
\hline & 3000 & 3.5 & 1.8 & 0.43 \\
\hline \multirow[t]{2}{*}{ Cha $\mathrm{H} \alpha 5, \mathrm{MJD}=51658.128$} & 3100 & 4.0 & 1.5 & 0.40 \\
\hline & 3000 & 3.5 & 1.5 & 0.40 \\
\hline \multirow[t]{2}{*}{ Cha $\mathrm{H} \alpha 6, \mathrm{MJD}=51658.143$} & 3100 & 4.0 & 1.8 & 0.44 \\
\hline & 2800 & 3.5 & 1.8 & 0.43 \\
\hline \multirow[t]{2}{*}{ Cha $\mathrm{H} \alpha$ 8, MJD = 51639.100} & 3100 & 4.0 & 1.5 & 0.40 \\
\hline & 2900 & 3.5 & 1.5 & 0.40 \\
\hline \multirow[t]{2}{*}{ Cha $\mathrm{H} \alpha$ 8, MJD $=51658.215$} & 3100 & 4.0 & 1.6 & 0.41 \\
\hline & 2900 & 3.5 & 1.7 & 0.42 \\
\hline
\end{tabular}

\begin{tabular}{cccc}
\hline \hline$T_{\text {eff }}$ & $\log (g)$ & $\log (\epsilon)$ & $\begin{array}{c}E W_{\mathrm{Li}} \\
(\mathrm{K})\end{array}$ \\
\hline Setup 2 & & & \\
\hline 3000 & 5.5 & 1.3 & 0.38 \\
2900 & 3.5 & 0.9 & 0.33 \\
3000 & 5.5 & 1.6 & 0.45 \\
2900 & 3.5 & 1.6 & 0.41 \\
3000 & 5.5 & 1.6 & 0.45 \\
2900 & 3.5 & 1.4 & 0.39 \\
\hline 3000 & 5.5 & 1.3 & 0.38 \\
2800 & 3.5 & 1.0 & 0.35 \\
3100 & 4.0 & 1.5 & 0.40 \\
2800 & 3.5 & 1.5 & 0.41 \\
3100 & 4.0 & 1.4 & 0.39 \\
2800 & 3.5 & 1.3 & 0.39 \\
\hline 3000 & 5.5 & 1.9 & 0.54 \\
3000 & 5.5 & 1.9 & 0.54 \\
3000 & 5.5 & 1.9 & 0.54 \\
3000 & 5.5 & 1.9 & 0.54 \\
3000 & 5.5 & 1.7 & 0.48 \\
3000 & 5.5 & 1.7 & 0.48 \\
3000 & 5.5 & 1.6 & 0.45 \\
3000 & 5.5 & 1.7 & 0.48 \\
3000 & 5.5 & 1.7 & 0.48 \\
3000 & 5.5 & 1.7 & 0.48 \\
3100 & 4.0 & 1.6 & 0.41 \\
3000 & 5.5 & 1.7 & 0.48 \\
3000 & 5.5 & 1.6 & 0.45 \\
3000 & 5.5 & 1.6 & 0.45 \\
3000 & 5.5 & 1.7 & 0.48 \\
3000 & 5.5 & 1.7 & 0.48 \\
\hline 3000 & 5.5 & 1.8 & 0.51 \\
3000 & 5.5 & 1.8 & 0.51 \\
3000 & 5.5 & 1.6 & 0.45 \\
3000 & 5.5 & 1.6 & 0.45 \\
\hline 3000 & 4.0 & 1.9 & 0.45 \\
2800 & 3.5 & 1.8 & 0.44 \\
\hline 3000 & 5.5 & 1.6 & 0.45 \\
2900 & 3.5 & 1.5 & 0.40 \\
3100 & 4.0 & 1.9 & 0.45 \\
2900 & 3.5 & 1.7 & 0.42 \\
\hline & & &
\end{tabular}

(see Fig. 1), this approach is problematic. Setting the continuum right is especially difficult for observations of relative low signalto-noise ratio, especially those of Cha $\mathrm{H} \alpha 2,3$ and 8. Another difference in the two determinations of the $v \sin i$ is that in the old data, the spectra were deconvolved with the point-spreadfunction (PSF) of the spectrograph, which was determined from the telluric lines. Given the differences in the two approaches, it is not surprising that the $v \sin i$-values obtained are slightly different from the older ones. Altogether, our empirical error estimate for $\log (\epsilon)$ is 0.4 dex. It is derived from studying the changes due to the variation of the free parameters.

In Table 2 we also list the values of the lithium $E W$ s. The most crucial step when determining the $E W$ is identifying the continuum in the spectrum; this is the main source of error, see the discussion in Sect. 1. The "true" continuum (ignoring the effects of all atomic and molecular lines in the spectrum) is at much higher flux levels than the observed pseudo-continuum. Therefore, we construct a pseudo-continuum in the wavelength range from $6700 \AA$ to $6720 \AA$ by calculating a spectrum without the lithium lines. After taking the difference between this pseudo continuum and the synthetic spectrum, the remaining area is normalized over the mean flux between the $\mathrm{D}_{1}$ and $\mathrm{D}_{2}$ line of Li I, resulting in the theoretical/synthetic $E W \mathrm{~s}$, which are also listed in Tables 2 and 3.

\section{Results}

The results are listed in Table 2 and are presented graphically in Figs. 2-7. For most objects more than one observed spectrum has been fitted. Figures 2-7 display for each object the model fit to one observation as an example. The upper panels in the figures show the fit over all free parameters, while the lower panels show the fit with fixed effective temperature. The relatively low signal-to-noise ratio of the observations limits the fit quality, in particular when applying the first fitting method with more free parameters. This is less severe for the case of Cha $\mathrm{H} \alpha 4$, for which the obtained spectra have a higher signal-to-noise ratio. 
Table 3. Physical parameters of BDs/VLMSs in Chamaeleon I derived from a comparison of synthetic GAIA-cond models and high-resolution by Natta et al. (2004) using different line profile setups: (from left to right) effective temperature, surface gravity, lithium abundance and $E W$ of lithium. The given results are for (i) a fit to the full range of free model parameters: $T_{\text {eff }}, \log (g), \log (\epsilon)$, radial velocity and rotational velocity (first row of each observation) and (ii) for a fit with fixed effective temperature as determined by Comerón et al. (2000) (second row).

\begin{tabular}{|c|c|c|c|c|}
\hline$\overline{\overline{\text { Object }}}$ & $\begin{array}{l}T_{\text {eff }} \\
(\mathrm{K})\end{array}$ & $\overline{\overline{\log (g)}}$ & $\overline{\log (\epsilon)}$ & $\begin{array}{c}E W_{\mathrm{Li}} \\
(\AA)\end{array}$ \\
\hline \multicolumn{5}{|c|}{ Setupl } \\
\hline \multirow[t]{2}{*}{ Cha $\mathrm{H} \alpha 2$} & 3100 & 4.0 & 1.2 & 0.37 \\
\hline & 2900 & 3.5 & 1.2 & 0.37 \\
\hline \multirow{2}{*}{ Cha $\mathrm{H} \alpha 3$} & 3100 & 4.0 & 1.3 & 0.38 \\
\hline & 2800 & 3.5 & 1.3 & 0.38 \\
\hline \multirow[t]{2}{*}{ Cha $\mathrm{H} \alpha 5$} & 3100 & 4.0 & 1.5 & 0.40 \\
\hline & 3000 & 3.5 & 1.5 & 0.40 \\
\hline \multirow[t]{2}{*}{ Cha $\mathrm{H} \alpha 6$} & 3100 & 4.0 & 1.6 & 0.41 \\
\hline & 2800 & 3.5 & 1.5 & 0.41 \\
\hline
\end{tabular}

\begin{tabular}{llll}
\hline \hline $\begin{array}{l}T_{\text {eff }} \\
(\mathrm{K})\end{array}$ & $\log (g)$ & $\log (\epsilon)$ & $\begin{array}{c}E W_{\mathrm{Li}} \\
(\AA)\end{array}$ \\
\hline $\begin{array}{l}\text { Setup2 } \\
3000\end{array}$ & 5.5 & 1.5 & 0.43 \\
2900 & 3.5 & 1.3 & 0.38 \\
\hline 3000 & 5.5 & 1.6 & 0.45 \\
2800 & 3.5 & 1.3 & 0.39 \\
\hline 3000 & 5.5 & 1.7 & 0.48 \\
3000 & 5.5 & 1.7 & 0.48 \\
\hline 3000 & 5.5 & 1.7 & 0.48 \\
2900 & 3.5 & 1.5 & 0.41 \\
\hline
\end{tabular}

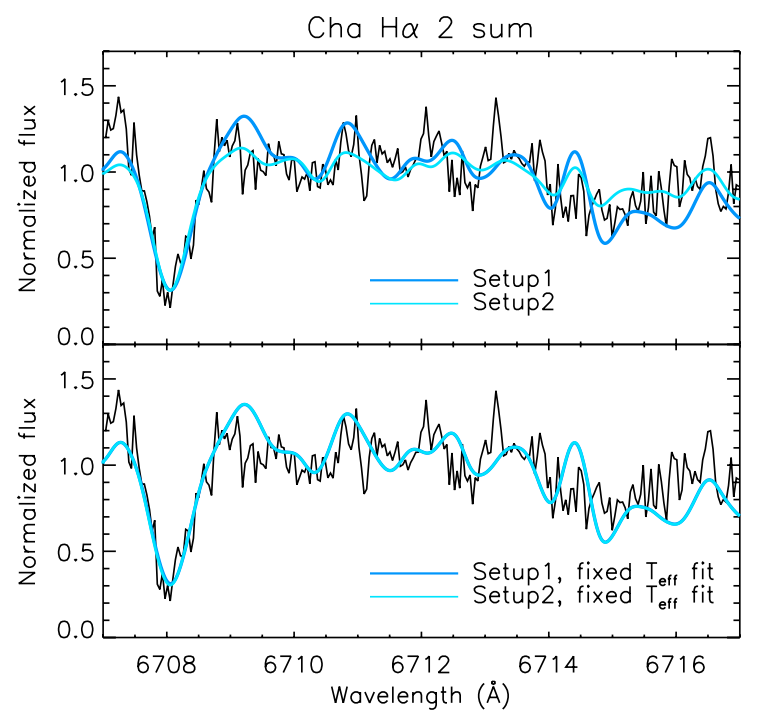

Fig. 2. High-resolution UVES spectrum of Cha $\mathrm{H} \alpha 2$ covering the lithium line at $6708 \AA$ (Joergens \& Guenther 2001; Joergens 2006) fitted with synthetic GAIA-cond models using 2 different line profile setups as described in Table 2. Upper panel: observation with free fit (first row of each observation in Table 2) and lower panel: observation with "effective temperature fixed fit" (second row of each observation in Table 2).

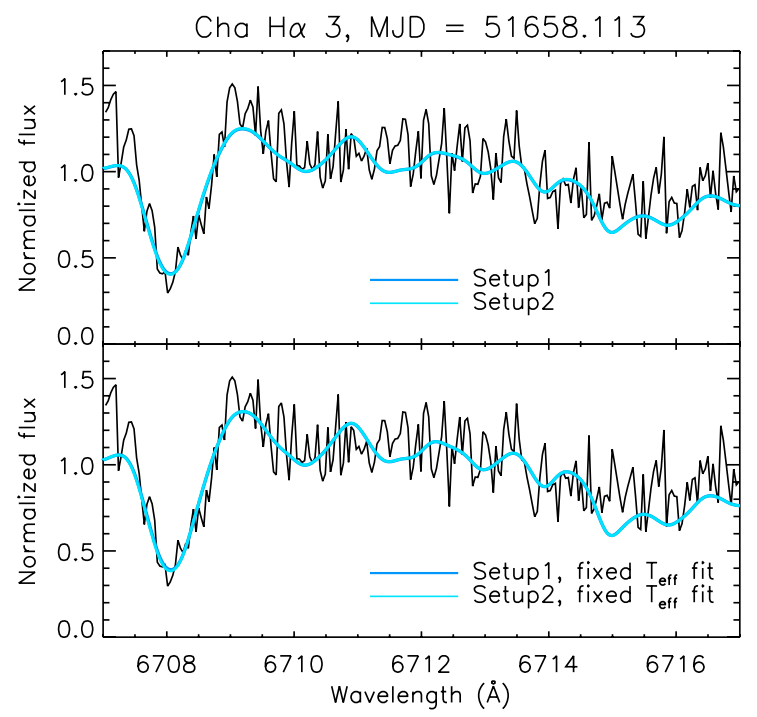

Fig. 3. The same as in Fig. 2 for the observation of $\mathrm{Cha} \mathrm{H} \alpha 3$.

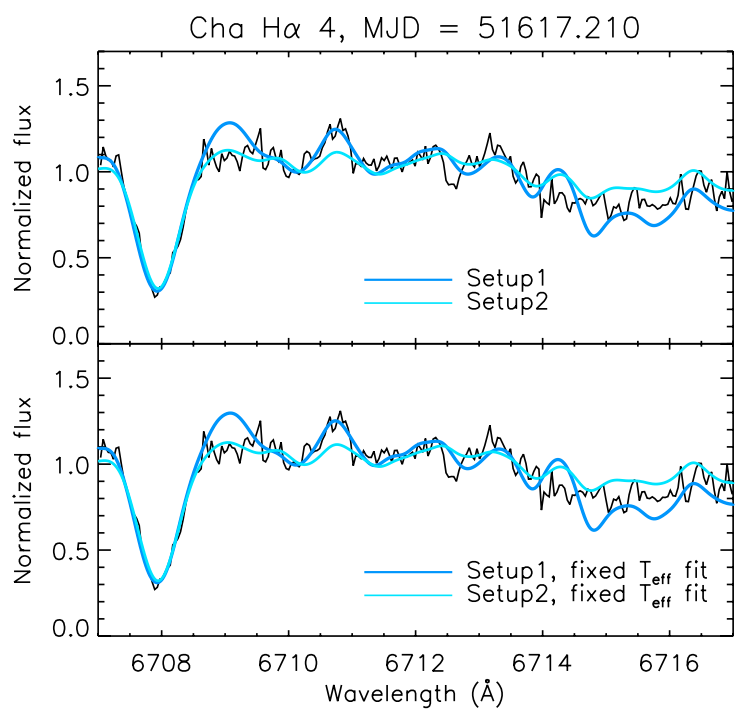

Fig. 4. The same as in Fig. 2 for the observation of $\mathrm{Cha} \mathrm{H} \alpha 4$.

In the following, we give details for the fits to the individual observations.

Due to the relatively low signal-to-noise ratios in the observations of Cha $\mathrm{H} \alpha 2$ and 3 (Fig. 3), we have added the two spectra of each of the objects (labeled with "sum"). An improved fitting with these spectra is possible. The fit results are consistent with the two methods and hint towards the higher lithium abundance. The description of Cha $\mathrm{H} \alpha 2$ is mostly satisfying, as depicted in Fig. 2. The blue and the red part of the lithium absorption line, as well as the red wing, are nicely delineated. However, there is a feature from approximately $6714.4 \AA$ to $6716 \AA$ in the synthetic spectra that cannot be identified through the observations. We assume that this feature is due to the TiO bands in the synthetic spectra, which cover this wavelength range. The red wing of the Li I line of $\mathrm{Cha} \mathrm{H} \alpha 3$ is described fairly well by the setups, in contrast, the fitting of the blue wing is unsatisfactory. This could be due to the very limited range of the observation at the shorter wavelengths.

The observations of Cha $\mathrm{H} \alpha 4$ have the best signal-to-noise ratio, therefore, a more detailed description is possible, as shown in Fig. 4. Both setups provide a good representation of the absorption core. The fits of Setup1 tend to show a feature at the beginning of the red wing, at approximately $6709 \AA$, contrary to the observations. Note the existence of a similar feature in the observation shown in Fig. 2, in which this feature is described by 


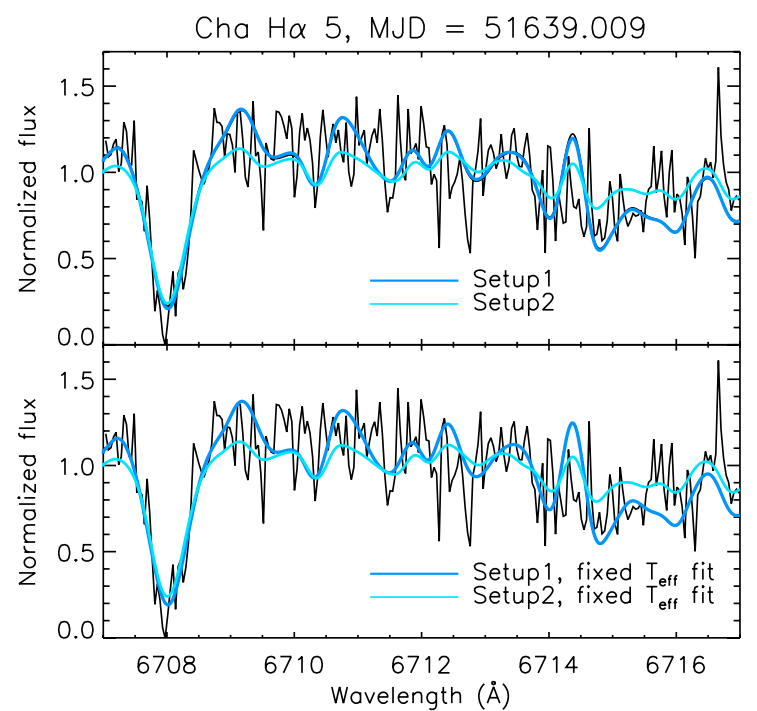

Fig. 5. The same as in Fig. 2 for the observation of $\mathrm{Cha} \mathrm{H} \alpha 5$.

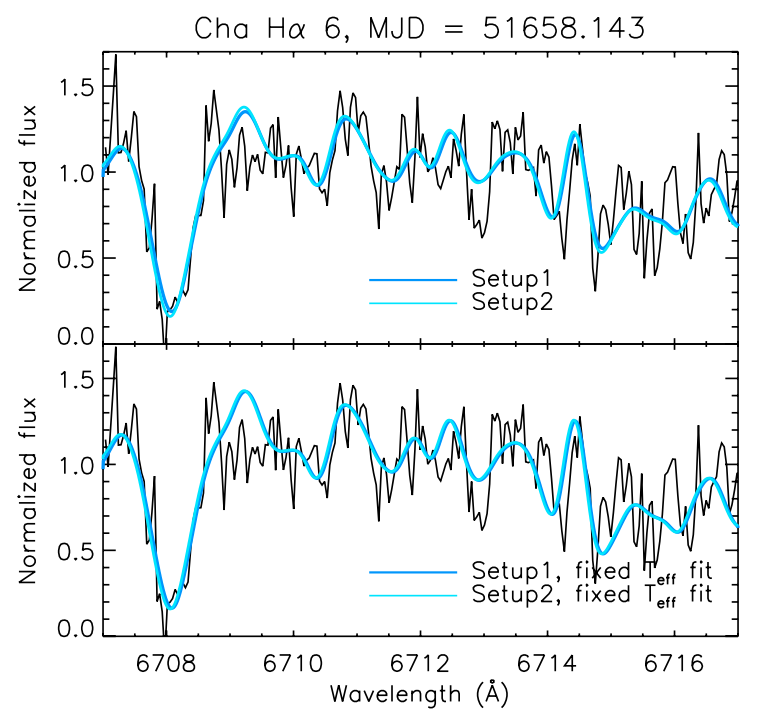

Fig. 6. The same as in Fig. 2 for the observation of Cha $\mathrm{H} \alpha 6$.

the synthetic spectra. The lack of this feature appears to be characteristic for Cha $\mathrm{H} \alpha$ 4. The feature at approximately $6710.7 \AA$ is well described. The fits of Setup2 provide a better description at $6709 \AA$, but an unsufficient one at $6710.7 \AA$, compared to the fit with Setup1. From Table 2 it can be seen that both fits result in an equal, or very similar, lithium abundance estimate for each setup. In particular, the fits for the synthetic spectra of Setup2 give the same effective temperature and surface gravity, except for one case, which is very close.

We consider in the following the effect of surface activity on the measured lithium abundances. The fluctuations in the derived lithium abundances (Table 2) for the different observations of Cha $\mathrm{H} \alpha 4$ are well within the estimated error of 0.4 dex in $\log (\epsilon)$. Studies of the variability of Cha $\mathrm{H} \alpha 4$, based on radial velocity monitoring (Joergens 2006; partly the very same spectra as evaluated here) and quasi-simultaneous photometric monitoring (Joergens et al. 2003) on the time scale of its rotational period (Joergens \& Guenther 2001), indicate a very small surface activity level for Cha $\mathrm{H} \alpha 4$. The determined photometric peak-to-peak amplitudes in the Bessel $R$ and Gunn $i$ filters $(\Delta R<0.03 \mathrm{mag}, \Delta i<0.03 \mathrm{mag})$ and the radial velocity

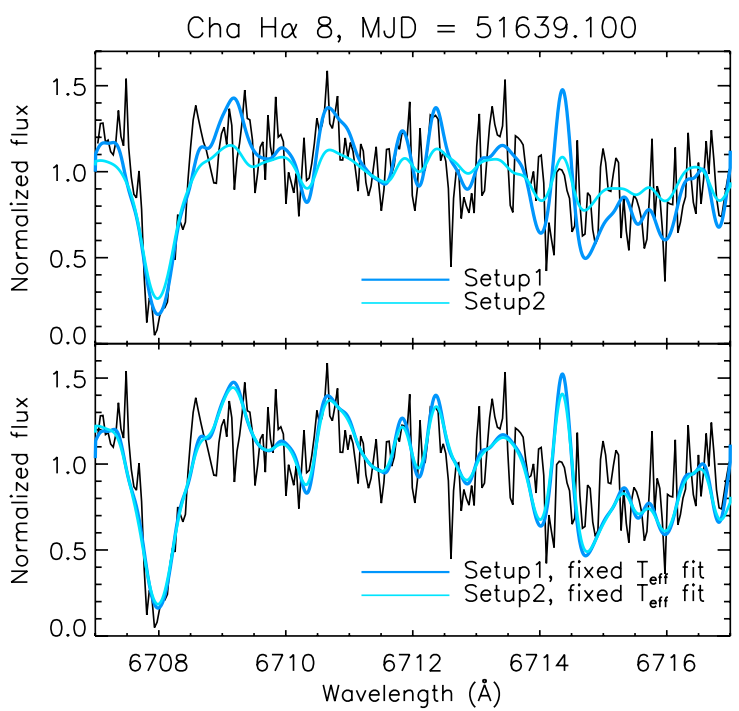

Fig. 7. The same as in Fig. 2 for the observation of Cha $\mathrm{H} \alpha 8$.

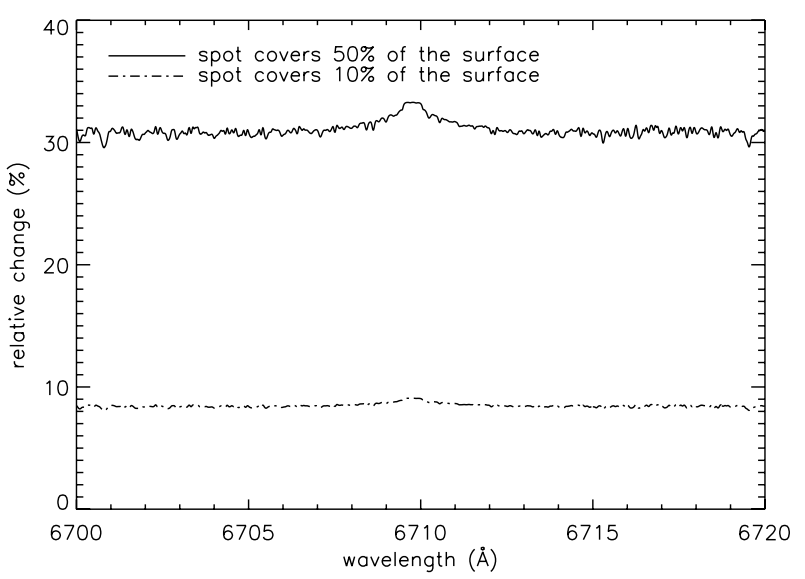

Fig. 8. Relative change in the spectrum, when assuming a $500 \mathrm{~K}$ cooler spot with a spatial coverage of $10 \%$ and $50 \%$ respectively.

peak-to-peak amplitude $\left(\Delta R V<0.2 \mathrm{~km} \mathrm{~s}^{-1}\right)$ allow us to estimate that if caused by a spot or spots $500 \mathrm{~K}$ cooler than the surrounding atmosphere, these cover less than $1 \%$ of its surface. However, for general interest, we consider here the influence of spots on the lithium abundance measurements. The problem with spots in $\mathrm{G}$ and $\mathrm{K}$ stars is that their contribution to the continuum is very small, so that $T_{\text {eff }}$ essentially comes from the spot free region since the Li I line becomes stronger in cooler atmospheres. Hence to a large part, the Li I absorption line would originate from the cool spot. Thus, the atmospheric model that is used to determine the abundance and $E W$ of the Li I line would have a wrong temperature. Such effects have been observed in very active $\mathrm{G}$ and $\mathrm{K}$ stars, like EK Draconis (König et al. 2005). For objects of spectral type $\mathrm{M}$ or later this effect should not play a role, because these objects are so cool that the Li I originates mainly from the photosphere. In order to demonstrate this, we assume a spot with an effective temperature which is $500 \mathrm{~K}$ cooler than a given effective temperature of the object, which is assumed to be $3100 \mathrm{~K}$. Furthermore, we assume that the spot coverage is $10 \%$ of the object. Although this is quite a substantial spot for such an object, the depth of the Li I line in the spectrum only changes by approximately a seven-tenth of a percent (dashed-dotted line in Fig. 8). Even when increasing the spot area to $50 \%$, the relative change is just $2.4 \%$ (solid line in Fig. 8), so that the effect 


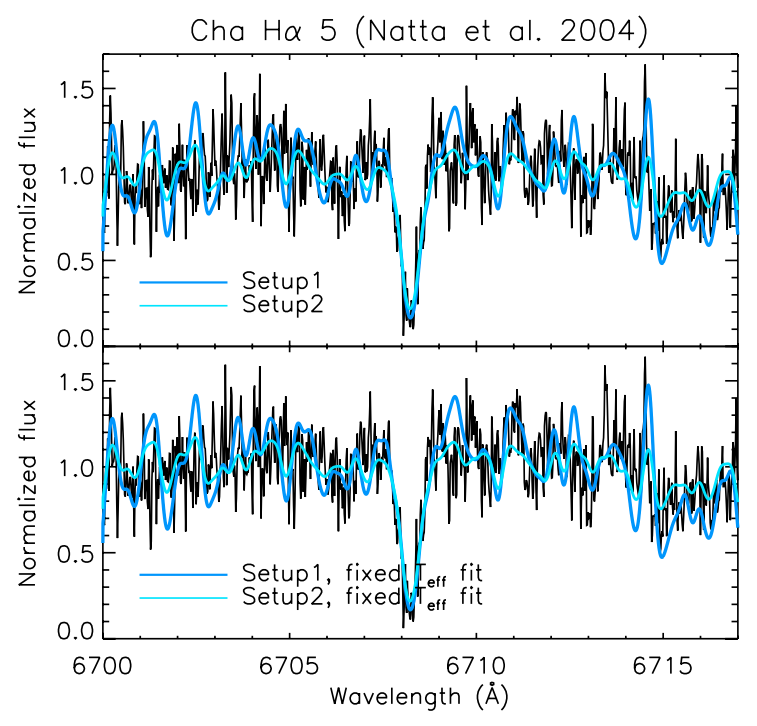

Fig. 9. The same as in Fig. 2 for the observation of Cha $\mathrm{H} \alpha 5$ using the spectra obtained by Natta et al. (2004).

of spots at the surface has a small effect on the spectrum of the lithium lines. We thus conclude that even very large spots have essentially no influence on the abundance in objects of spectral type $\mathrm{M}$.

In Figs. 5 and 7 the results of the fitting procedure are displayed for Cha $\mathrm{H} \alpha 5$ and 8. With the fits of Setup1 and the "effective temperature fixed fits" for Setup2, a satisfactory fit quality is reached. The free fit of Setup2 underestimates the flux especially towards the blue wing, but nevertheless gives a reasonable description of the pseudo-continuum. For Cha $\mathrm{H} \alpha$ 6, all three setups are in agreement and provide a sufficient description, see Fig. 6.

When fitting the observations of Cha $\mathrm{H} \alpha 2,3,5$ and 6 by Natta et al. (2004) similar results to the previous ones are obtained. Their observations have the same resolution but a better coverage of the Li I absorption line. The same fitting procedure was applied to these observations. In Table 3, the results are summarized. In Fig. 9, representing $\mathrm{Cha} \mathrm{H} \alpha$, the results with the free fit and fixed effective temperature fit are shown. The fits have been performed for both the wavelength range that has been used before and additionally the one from $6700 \AA$ to $6717 \AA$. The fit results are not wavelength range dependent. For Cha $\mathrm{H} \alpha 2,3$ and 5 good fits were possible, however the $\mathrm{Li}$ I line core of Cha $\mathrm{H} \alpha 6$ could be fitted only very poorly. Nevertheless, the line wing and pseudo continuum can be described satisfactorily, also for $\mathrm{Cha} \mathrm{H} \alpha 6$.

The effective temperatures for Cha $\mathrm{H} \alpha 4$ and Cha $\mathrm{H} \alpha 5$ (plus $\mathrm{Cha} \mathrm{H} \alpha 2$ for Setup2 and Cha $\mathrm{H} \alpha 8 \mathrm{MJD}=51639.100$ for Setup2) derived from our model fits are consistent within the error estimate of $\pm 100 \mathrm{~K}$ in both setups with the effective temperatures from Comerón et al. (2000), as can be seen in Table 2. For the other objects there is a greater deviation of an additional 100-200 K (we assume improvements after narrowing down the free fit parameters in future work). When comparing the derived effective temperatures in Table 2 with those of Luhman (2004), the agreement is very good for all objects fitted with every setup, since he provides slightly higher effective temperatures. The values of the derived surface gravities depend strongly on the used setup. The fit with Setup2 provides higher surface gravities, often at the upper limit. When applying Setup1, the free fit provides a surface gravity within its range, whereas the

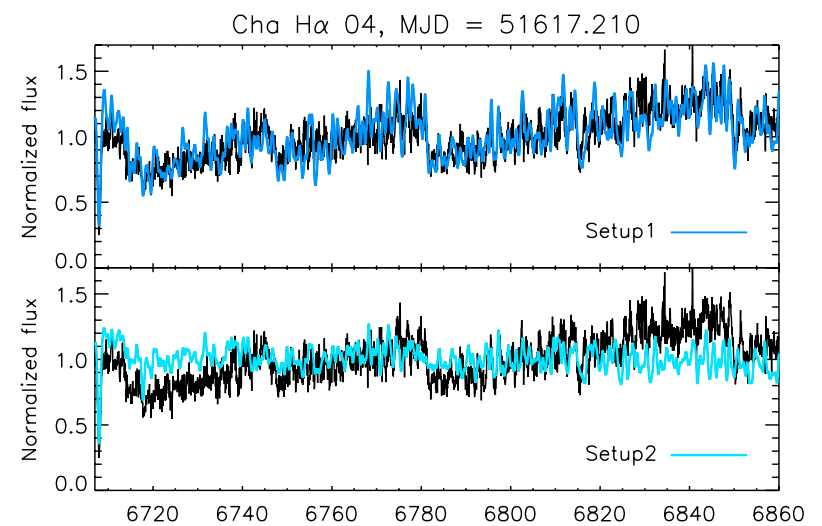

Fig. 10. The $\mathrm{TiO}$ bands are depicted for $\mathrm{Cha} \mathrm{H} \alpha 4, \mathrm{MJD}=51617.210$ with the free fit with both setups.

"effective temperature fixed fit" always results in the lowest surface gravity value. Chabrier \& Baraffe (2000) predict for substellar objects of ages between one Myr and $10 \mathrm{Myr} \operatorname{ag} \log (g)$ around 3.5 and 4.0. Hence, these low values of $\log (g)$ also imply the relatively young age of Chamaeleon I. Consequently, we want to stress that no final statement about the surface gravity can be made yet by fitting the Li I absorption line. Further studies for obtaining the surface gravity are necessary and will be performed in future work. Nevertheless, both fit possibilities, the "effective temperature fixed fit" and the free fit, yield very similar values of the lithium abundance, independent of the effective temperature. When comparing the lithium abundance results between the two setups there is just a small visible discrepancy, which is consistent with the results within the estimated errors (Sect. 3). The mean values of the derived lithium abundances is 1.5 for Setup1 and 1.6 for Setup2. However, our tests show that the derived parameters provide a good description of the overall continuum of the observations, e.g., the continuum characteristic and the $\mathrm{TiO}$ bands. These bands are shown in Figs. 10-13, representing Cha $\mathrm{H} \alpha 4$, MJD = 51 617.210, and Cha $\mathrm{H} \alpha 6, \mathrm{MJD}=51658.143$. The fits have been normalized via their medians in this wavelength range and do not show large differences between the two fitting procedures. When comparing the EWs derived by Joergens \& Guenther (2001) with those presented in Table 2, we find that they are mostly in agreement, within their error range. For Cha $\mathrm{H} \alpha 2$ only the values of the first spectrum are in agreement. Generally our $E W$ s of Cha $\mathrm{H} \alpha 3$ and 6 are greater (or at the upper limit within the error bars of Joergens \& Guenther 2001). The values of Cha $\mathrm{H} \alpha 4,5$ and 8 are consistent with the ones of Joergens \& Guenther (2001). The reason for this slight discrepancy is due to the different methods with which the EWs are obtained, see the discussion in Sect. 1 about the difficulties when trying to obtain the $E W \mathrm{~s}$.

The Li I line in the first data set is in the last order close to the edge of our spectra range. Using a different setting, Natta et al. (2004) have obtained spectra of Cha $\mathrm{H} \alpha 2,3,5$ and 6 using a setting in which the Li I line is in the middle of the spectrum. We have analyzed both data sets and have basically found the same low lithium abundances in both cases, see Tables 2 and 3 for details.

We compare the here derived lithium abundances for objects close to the substellar border (M6-M7) with those of higher mass Chamaeleon I members (Sz 19, Sz 6, Sz 24, Sz 3, Sz 5 and Sz 9) determined by Magazzu et al. (1992). Magazzu et al. (1992) classify them as classical T Tauri stars of spectral type G2, K2, and M0. They are relatively young, below 0.3 to $1.3 \mathrm{Myr}$, which 


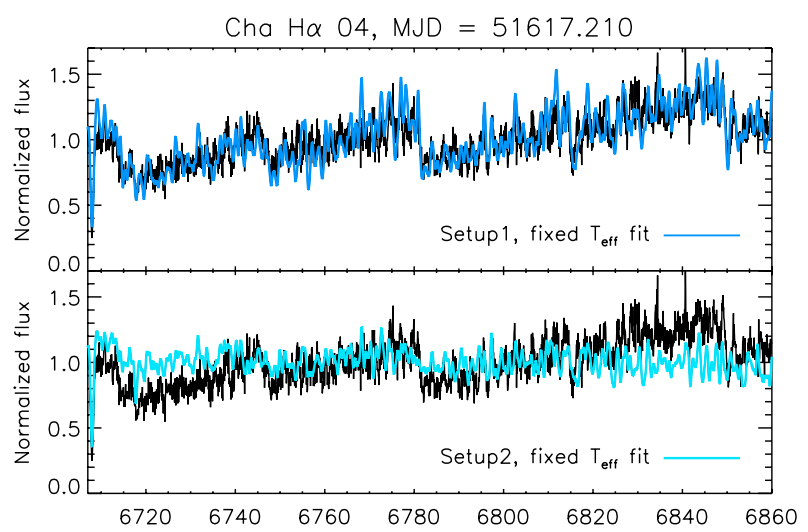

Fig. 11. The $\mathrm{TiO}$ bands are depicted for $\mathrm{Cha} \mathrm{H} \alpha$ 4, $\mathrm{MJD}=51617.210$ with the "fixed effective temperature fit" setups.

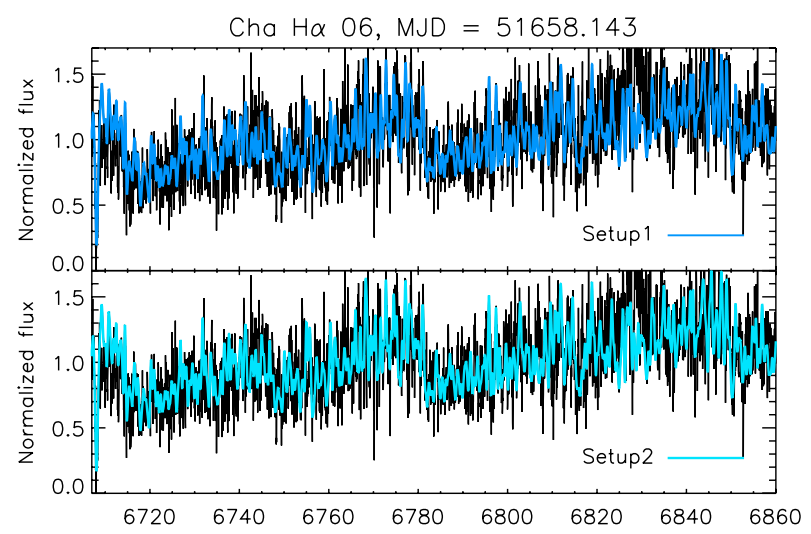

Fig. 12. The $\mathrm{Ti} \mathrm{O}$ bands are depicted for $\mathrm{Cha} \mathrm{H} \alpha 6, \mathrm{MJD}=51658.143$ with the free fit with both setups.

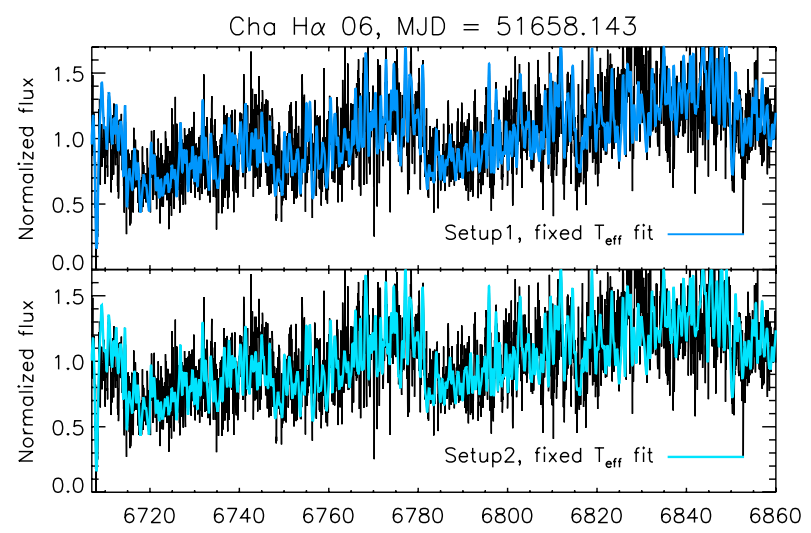

Fig. 13. The $\mathrm{TiO}$ bands are depicted for $\mathrm{Cha} \mathrm{H} \alpha 6, \mathrm{MJD}=51658.143$ with the "fixed effective temperature" fit.

confirms the discussion regarding the young age of these cloud members given in the introduction. Moreover, it is assumed that there is no lithium depletion in the sample of the classical T Tauri stars.

Magazzu and colleagues derive the lithium abundances via curves of growth obtained from LTE- and nonLTE-calculations and compare them to observed $E W$ s after correcting for veiling. For the six classical T Tauri stars mentioned above they obtain a mean $\log (\epsilon)$ of 3.1 from LTE-calculations and 3.4 from non-LTE-calculations, which are values much closer to the meteorite lithium abundance.
Classical T Tauri stars show a strong infrared-excess due to the presence of an accretion disk. In addition to the infraredexcess, classical T Tauri stars often have photospheric absorption lines, which have smaller $E W \mathrm{~s}$ than main sequence stars of similar spectral types. This is generally attributed to the presence of an additional continuum and perhaps an emission line source, which is interpreted as the signature of the accretion shock on the surface of the stars (see Hessman \& Guenther 1997 for details). Since young BDs are also known to have accretion disks (Natta et al. 2004; Mohanty et al. 2005), it is possible that our spectra of the young BDs are effected by such a veiling continuum. Jayawardhana et al. (2003) have in fact detected an $L$-band excess for our $\mathrm{Cha} \mathrm{H} \alpha$ objects and estimate a disk fraction of 40-60\% for objects in Chamaeleon. In addition, Persi et al. (2000) have observed 108 stars and BDs in Chameleon at 6.7 and $14.3 \mu \mathrm{m}$, of which 82 sources show infrared-excess. Although most of these objects are classical T Tauri stars, some are BDs. The largest excess of a BD was found in Cha $\mathrm{H} \alpha 2$. It can thus be concluded that disks are also common amongst the low-mass objects studied in this work. Hence the question arises as to whether low-mass objects could also have a veiling continuum, in analogy to the $\mathrm{T}$ Tauri stars. The veiling continuum of T Tauri stars originates from an accretion shock on the surface of the star, which forms because of a gap between the disk and the star. One would expect that the temperature of the shock in objects of lower mass would be correspondingly lower. Whether objects of very low-mass have shocks of sufficiently high temperature to veil the optical continuum is an open question that can only be addressed observationally. White \& Basri (2003) studied three very low-mass objects of spectral type M7 in Taurus, and conclude that while these objects do show clear signs of disks and accretion, there is no detectable veiling continuum. Using our spectra, we also investigated whether the spectra are veiled or not by using the $\mathrm{TiO}$-lines. If the spectra are veiled, $\mathrm{TiO}$-lines in the model would have to be stronger than the observed ones. As shown in Figs. 10-13, this is not the case, as we are able to fit the $\mathrm{TiO}$-lines with the $\log (g)$ and $T_{\text {eff }}$-values listed in Table 2 without assuming the presence of a veiling continuum. We therefore conclude that the veiling continuum is not very prominent in these objects.

An explanation for the discrepancies in the derived lithium abundances between the BDs/VLMSs studied here at the substellar boundary and higher mass $\mathrm{T}$ Tauri stars studied by Magazzu et al. (1992), could be an error in the estimate of age and mass, not only because of the uncertainties in the evolutionary track predictions below $10 \mathrm{Myr}$, but also because of uncertainties in the measurement of the luminosity and effective temperatures, which have been used in the comparison with the evolutionary tracks, see Table 1 . The critical question is whether the observed large depletion of the lithium can be explained by the objects being either older or more massive than assumed. This is possible because the uncertainties in the determination of the ages of these objects are in fact large (see Table 1). For example, Comerón et al. (1999) finds a mean age of roughly $0.5 \mathrm{Myr}$ for our objects, whereas Comerón et al. (2000) gives ages between 2 and 5.5 Myr. However, these age estimates for Cha $\mathrm{H} \alpha \mathrm{BD}$ candidates and VLMSs are still in general agreement with the young age of the Cha I association, as indicated by the presence of classical T Tauri stars. When using the effective temperatures and luminosities derived by Luhman (2004), with the evolutionary tracks used by Comerón et al. (2000), the age and mass estimates again differ from the previous results. In order to test this hypothesis, we determined the ages and masses that the objects should have to show a lithium depletion like the 
one observed. According to Chabrier \& Baraffe (2000), the minimum age for the lithium depletion is $10 \mathrm{Myr}$ for an object of $0.1 M_{\odot}$. At younger ages the core temperatures are too low for the depletion of lithium. For objects of even lower masses, for example $0.07 M_{\odot}$, the age would have to be larger than $10^{7.7} \mathrm{yrs}$. For objects of higher mass, the lithium depletion starts at an earlier age. For example, at $0.3 M_{\odot}$ the depletion sets in at $10^{6.7} \mathrm{yrs}$. Thus, if the objects studied here had such a high mass, the observed lithium abundance would be explained. However, all evolutionary track calculations (Burrows et al. 1997; D’Antona \& Mazzitelli 1997; Baraffe et al. 1998) give much lower masses for our objects. Although the ages of the objects are uncertain, in none of the cases are the masses and ages sufficiently high to explain the lithium depletion when using the evolutionary tracks (Chabrier \& Baraffe 2000), regardless of whether we use the masses and ages from Comerón et al. (1999), Comerón et al. (2000), or Luhman (2004).

In summary, as possible explanations for the observed lithium depletion we can rule out the presence of a veiling continuum, the effects of spots, and that the objects are more massive or much older than previously thought. We are therefore left with two possible explanations: one is just a general lack of the understanding of the details of the formation of the lithium line, and the other is that the internal structure of the object differs from the internal structure of the evolutionary tracks predicted. This is possible because the objects are quite young and the evolutionary tracks may not have fully forgotten the initial conditions with which they were started. This could also be the case for the classical T Tauri stars from Magazzu et al. (1992), which could no longer be fully convective. As a result, they could have already started the destruction of lithium without displaying any evidence at the surface.

\section{Conclusions}

The lithium resonance lines in high-resolution spectroscopic observations of very young BDs and VLMSs in Cha I are fairly well described by the synthetic spectra calculated with the GAIA-cond atmosphere model using different model setups. The same is valid for the red pseudo-continua in the observations. We present the first study of the lithium abundance of these objects. The results of the lithium abundance obtained by the observations of Natta et al. (2004) confirm those observed by Joergens \& Guenther (2001). The synthetic spectra lead to similar results for the different line profile setups. However, there are small differences in the derived lithium abundance, as expected. The lithium abundances using Setup2 are generally the largest. In some cases, Setup1 provides a better description than Setup2, although Setup2 includes the detailed physics. However, next to the low resolution, the effective temperature range of our observed objects is near the upper limit for which the effect of pressure broadening described with van der Waals broadening is observable. A stronger statement is expected in an analysis of objects with later spectral type. Nevertheless, the results give a consistent picture and are within the estimated errors. Note that the $E W \mathrm{~s}$ presented here are synthetic ones. The choice of continuum has a large influence on their values. We have used pseudo synthetic continua assuming total lithium depletion.

Despite the young age of the studied BDs and VLMSs in Chamaeleon I and the assumption that lithium depletion has not yet started, the lithium abundances show an unexpected underabundance compared to meteoritic lithium abundance. We do not expect veiling to be an important effect for our objects. Observations with a higher resolution in the optical wavelength regime are important and needed for improved insight into the physics, including, e.g., the description of the more abundant alkalis, such as sodium and potassium, and the presence of satellites. Furthermore, a consistent set of observations of objects with spectral type $\mathrm{G}$ to $\mathrm{M}$ in $\mathrm{Cha} \mathrm{I}$ is desirable for an improved study with consistent model atmospheres and synthetic spectra.

More detailed theoretical models are necessary to evaluate if lithium depletion has already started in these objects, although this is a very difficult task.

Acknowledgements. We thank G. Torres for providing the estimate of the spot coverage for Cha $\mathrm{H} \alpha 4$ based on our photometric and radial velocity data. Furthermore, we thank A. Natta and S. Randich for making the spectra available.

\section{References}

Allard, F., \& Hauschildt, P. H. 1995, ApJ, 445, 433

Allard, F., Hauschildt, P. H., Alexander, D. R., Tamanai, A., \& Schweitzer, A. 2001, ApJ, 556, 357

Allard, N. F., Allard, F., \& Kielkopf, J. F. 2005, A\&A, 440, 1195

Allard, N. F., Allard, F., Johnas, C. M. S., \& Kielkopf, J. F. 2006, A\&A, submitted

Baraffe, I., Chabrier, G., Allard, F., \& Hauschildt, P. H. 1998, A\&A, 337, 403

Basri, G. 2000, ARA\&A, 38, 485

Burrows, A., Marley, M., Hubbard, W. B., et al. 1997, ApJ, 491, 856

Chabrier, G., \& Baraffe, I. 2000, ARA\&A, 38, 337

Chabrier, G., Baraffe, I., Allard, F., \& Hauschildt, P. H. 2000, ApJ, 542, 464

Comerón, F., Rieke, G. H., \& Neuhäuser, R. 1999, A\&A, 343, 477

Comerón, F., Neuhäuser, R., \& Kaas, A. A. 2000, A\&A, 359, 269

D’Antona, F., \& Mazzitelli, I. 1997, Mem. Soc. Astron. It., 68, 807

Gray, D. F. 1992, Science, 257, 1978

Hauschildt, P. H., \& Baron, E. 1999, JCAM, 109, 41

Hessman, F. V., \& Guenther, E. W. 1997, A\&A, 321, 497

Jayawardhana, R., Ardila, D. R., Stelzer, B., \& Haisch, Jr., K. E. 2003, AJ, 126, 1515

Joergens, V. 2006, A\&A, 446, 1165

Joergens, V., \& Guenther, E. 2001, A\&A, 379, L9

Joergens, V., Fernández, M., Carpenter, J. M., \& Neuhäuser, R. 2003, ApJ, 594, 971

Johnas, C. M. S., Allard, N. F., Homeier, D., Allard, F., \& Hauschildt, P. H. 2006, AIP Conf. Proc., 874, 354

Kenyon, M. J., Jeffries, R. D., Oliveira, J. M., \& Maxted, P. F. L. 2005, MNRAS, 356,89

König, B., Guenther, E. W., Woitas, J., \& Hatzes, A. P. 2005, A\&A, 435, 215

López Martí, B., Eislöffel, J., Scholz, A., \& Mundt, R. 2004, A\&A, 416, 555

Luhman, K. L. 2004, ApJ, 602, 816

Magazzu, A., Rebolo, R., \& Pavlenko, I. V. 1992, ApJ, 392, 159

Mohanty, S., Jayawardhana, R., \& Basri, G. 2005, ApJ, 626, 498

Natta, A., Testi, L., Muzerolle, J., et al. 2004, A\&A, 424, 603

Neuhäuser, R., \& Comerón, F. 1999, A\&A, 350, 612

Pavlenko, Y. V. 2005, Astronomische Nachrichten, 326, 934

Pavlenko, Y., Zapatero Osorio, M. R., \& Rebolo, R. 2000, A\&A, 355, 245

Persi, P., Marenzi, A. R., Olofsson, G., et al. 2000, A\&A, 357, 219

Rebolo, R., Martin, E. L., \& Magazzu, A. 1992, ApJ, 389, L83

Schweitzer, A., Gizis, J. E., Hauschildt, P. H., Allard, F., \& Reid, I. N. 2001, ApJ, 555, 368

Schweitzer, A., Hauschildt, P. H., Allard, F., \& Basri, G. 1996, MNRAS, 283, 821

Tinney, C. G. 1998, MNRAS, 296, L42

Tsuji, T. 2001, in Ultracool Dwarfs: New Spectral Types L and T, ed. H. R. A. Jones, \& I. A. Steele, 9

White, R. J., \& Basri, G. 2003, ApJ, 582, 1109

Zapatero Osorio, M. R., Béjar, V. J. S., Martín, E. L., et al. 2002, ApJ, 578, 536 